2021 in Hong Kong were identified. The primary endpoint was a composite of intensive care unit admission, use of invasive mechanical ventilation, and/or death. PPI user was identified by PPI use within 12 months before the diagnosis of COVID-19. In subgroup analysis, current PPI users were defined as patients who used PPIs within 1 month before the diagnosis of COVID-19; past PPI users were defined as patients who used PPIs 1 to 12 months before COVID-19 diagnosis. We performed sensitivity analysis after excluding patients with short-term new NSAID use within 1 month before COVID-19 diagnosis to minimize reverse causation bias.

Results We identified 8,675 COVID-19 patients (mean age 46 years, $49 \%$ male, $97.6 \%$ of all the reported cases in Hong Kong); 579 (6.7\%) patients had used PPI. PPI users were older, more likely to have comorbidities, concomitant medications and unfavorable laboratory parameters than non-users. Of 8,675 COVID-19 patients, 500 (5.8\%) developed the primary endpoint. After propensity score (PS) balancing for patients' demographics, comorbidities, laboratory parameters, and use of medications, PPI use was not associated with the development of primary endpoint in PS weighting (weighted hazard ratio [HR] 1.11, 95\% confidence interval [CI] $0.83-1.47, P=0.482$ ) (IDDF2021-ABS0122 Figure 1. Cumulative incidence of primary endpoint (a composite endpoint of intensive care unit [ICU] admission, use of invasive mechanical ventilation [IMV], and death) in COVID-19 patients who were and were not proton-pump inhibitor (PPI) users after propensity score (PS) weighting in a single multiple imputation data set.), and PS matching analysis (weighted HR 0.81, 95\%CI 0.57-1.14, $P=0.228$ ) (IDDF2021-ABS-0122 Figure 2. Cumulative incidence of primary endpoint (a composite endpoint of intensive care unit [ICU] admission, use of invasive mechanical ventilation [IMV], and death) in COVID-19 patients who were and were not proton-pump inhibitor (PPI) users after propensity score (PS) matching in a single multiple imputation data set). Consistent non-association was observed after

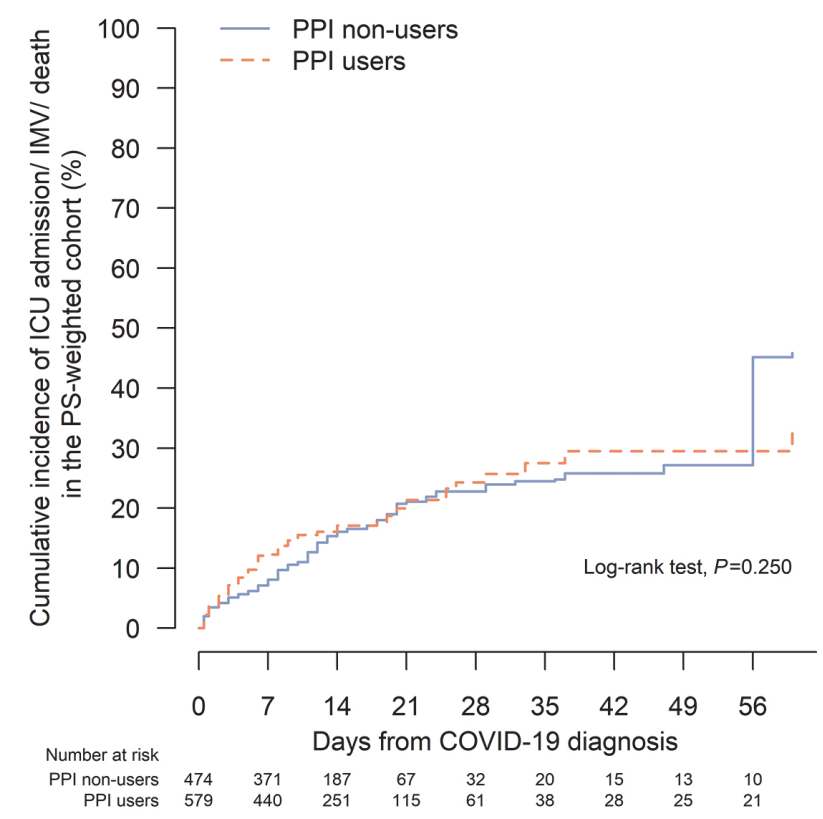

Abstract IDDF2021-ABS-0122 Figure 1

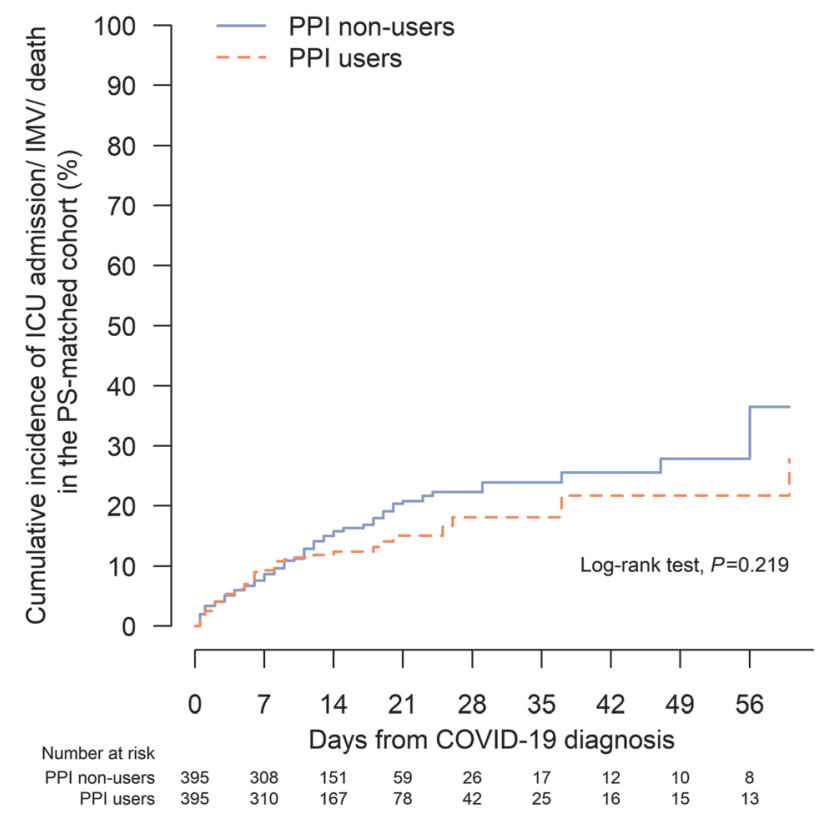

Abstract IDDF2021-ABS-0122 Figure 2

multivariable adjustment (adjusted HR 0.84, 95\%CI $0.66-$ $1.07, P=0.151)$, in subgroups of current and past PPI users, and in sensitivity analysis after excluding short-term new NSAID users.

Conclusions PPI use is not associated with adverse clinical outcomes in COVID-19 patients. The result remains robust after PS weighting, PS matching, multivariable adjustment, and subgroup analyses.

\section{Clinical Hepatology}

IDDF2021-ABS-0046
METABOLIC DYSFUNCTION-ASSOCIATED
FATTY LIVER DISEASE INCREASES COLON
CANCER RISK: A NATIONWIDE COHORT
STUDY

Hye Won Lee*, Seung Up Kim. Yonsei University College of Medicine, Korea, South

\subsection{6/gutjnl-2021-IDDF.14}

Background The association between nonalcoholic fatty liver disease (NAFLD) and colorectal cancer (CRC) has been controversial. Using the new consensus-driven definition, we evaluated the association of metabolic dysfunction-associated fatty liver disease (MAFLD) with the risk of developing CRC.

Methods From a nationwide health screening database, we included 8,933,017 participants (48.6\% male) aged 40-64 years between 2009 and 2010. Participants were categorised by the presence of fatty liver disease (FLD) - NAFLD and MAFLD, separately - and by the combination of the two definitions: Neither-FLD, NAFLD-only, MAFLD-only, or Both-FLD. The primary outcome was the development of CRC.

Results Among the participants, 2,517,330 (28.2\%) had NAFLD and 3,337,122 (37.4\%) had MAFLD, while 2,465,151 (27.6\%) met both NAFLD and MAFLD definitions. Over a median follow-up period of 10.1 years, 60,888 new 
CRC cases developed. NAFLD and MAFLD were each associated with a significantly higher risk of developing CRC. When the Neither-FLD group was the reference, multivariableadjusted hazard ratios (95\% confidence interval) for CRC were $1.16(1.06-1.28)$ in the NAFLD-only group, 1.18 (1.16$1.20)$ in the Both-FLD group, and $1.32(1.28-1.35)$ in the MAFLD-only group. The presence of advanced liver fibrosis further increased CRC risk in each FLD group.

Conclusions FLD was associated with a higher risk of CRC development. CRC risk was higher in the presence of MAFLD, especially when accompanied by liver fibrosis

\section{IDDF2021-ABS-0137 THE AMAP SCORE PREDICTS RISK OF HEPATOCELLULAR CARCINOMA DEVELOPMENT IN PATIENTS WITH ALCOHOL-RELATED CIRRHOSIS}

${ }^{1}$ Ken Liu*, ${ }^{2}$ Steven Masson, ${ }^{3}$ Waleed Fateen, ${ }^{3}$ Guruprasad Aithal, ${ }^{4}$ Terry CF Yip, ${ }^{5}$ Devanshi Seth. ${ }^{1}$ AW Morrow Gastroenterology and Liver Centre, Sydney, NSW, Australia; ${ }^{2}$ Faculty of Medical Sciences, Newcastle University Medical School, Framlington Place, Newcastle upon Tyne, UK; ${ }^{3}$ NIHR Nottingham Biomedical Research Centre, Nottingham University Hospitals and the University of Nottingham, Nottingham, UK; ${ }^{4}$ Department of Medicine and Therapeutics, Medical Data Analytic Centre, and Institute of Digestive Disease, The Chinese University of Hong Kong, Hong Kong; ${ }^{5}$ Centenary Institute of Cancer Medicine and Cell Biology, The University of Sydney, Sydney, Australia

\subsection{6/gutjnl-2021-IDDF.15}

Background The aMAP score (derived from age, male sex, albumin and platelet count) was recently devised to predict hepatocellular carcinoma (HCC) development. It was shown to have excellent performance irrespective of aetiology and ethnicity. Only one cohort of non-viral hepatitis patients (Japanese with mostly non-alcoholic fatty liver disease) was included in the validation set. Hence, the performance of the aMAP score among Caucasians with alcohol-related cirrhosis (ALC) is unknown. We aimed to validate the aMAP score in a cohort of ALC patients.

Methods Participants with ALC from three centres enrolled to a genome-wide association study (Schwantes-An et al. Hepatology 2021) were included. All participants had a history of high alcohol consumption. Cirrhosis was defined clinically (portal hypertension or decompensation), using Fibroscan and/ or histology. Patients were followed until last liver imaging, HCC development, liver transplantation, or death with the latter two adjusted as competing risks.

Results 270 ALC patients were included: male (72.2\%), Caucasian (98.9\%), with median age of 56 years, and compensated cirrhosis (median Child-Pugh score 5). The median aMAP score was 60: 12.6\% low-risk (score 0-50), $35.2 \%$ medium-risk (50-60) and 52.2\% high-risk (>60). After a median follow-up of 41 months, 13 patients developed HCC, 25 received liver transplantation and 106 died. The aMAP score predicted HCC development (hazard ratio 1.11, 95\% confidence interval 1.05-1.18, $P<0.001$, IDDF2021-ABS0137 Figure 1). The C-index for the aMAP score for predicting HCC development was $0.83,0.78$ and 0.80 at one, three and five years respectively which was similar to ADRESSHCC (Flemming et al. Cancer. 2014) and Ioannou et al.'s (J Hepatol. 2019) scores (IDDF2021-ABS-0137 Figure 2A-C). We then examined the predictive value of three genome-wide association-significant risk-increasing alleles (PNPLA3: rs2294915, TM6SF2:rs10401969, HSD17B13:rs10433937) using a genetic risk score. A three-gene score did not predict HCC development at five years (C-index 0.53) or improve the aMAP score performance when combined.

Conclusions We validated the excellent performance of the aMAP score in a cohort of ALC patients and affirmed its applicability across wider aetiologies.

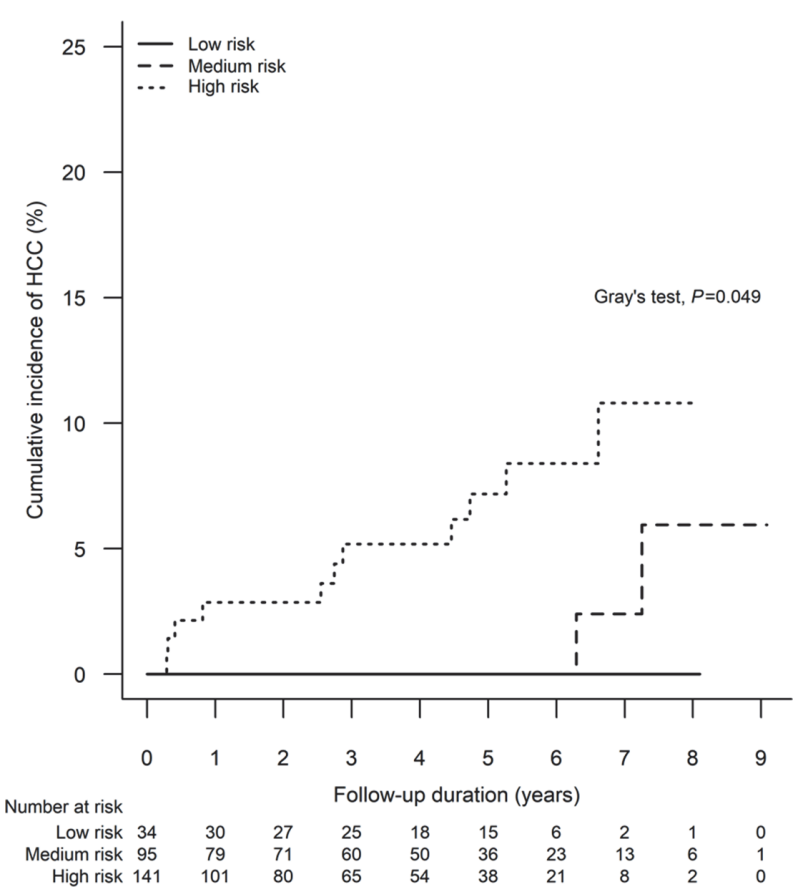

Abstract IDDF2021-ABS-0137 Figure 1
A

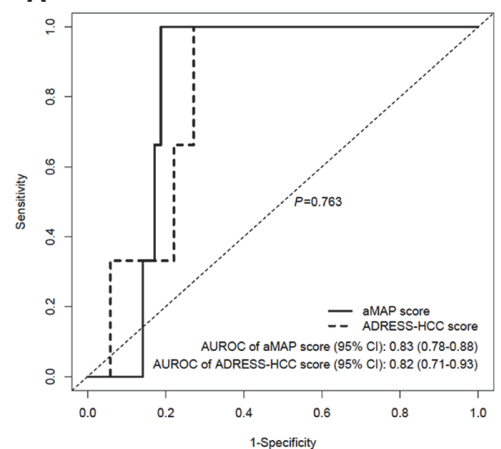

B

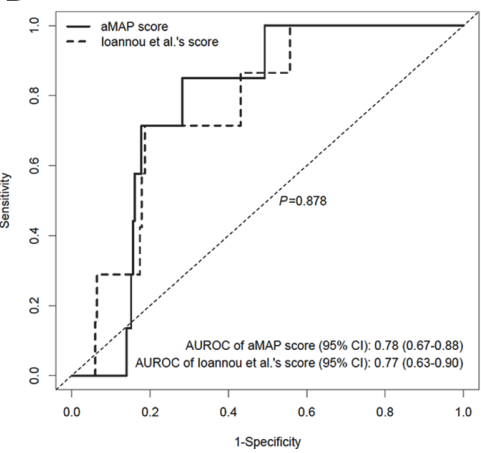

C

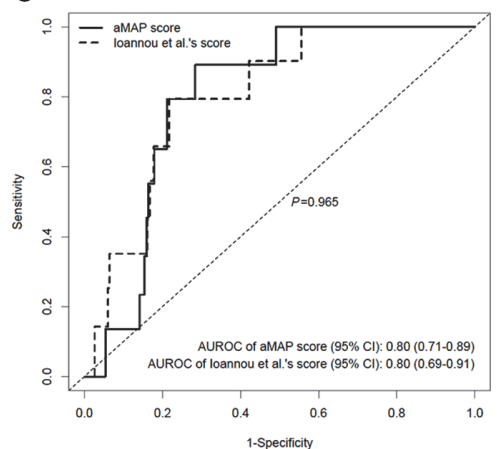

